\title{
Quantum Dot-in-a-Well (QDWELL) Laser Dynamics under Optical Injection
}

\author{
Y. Ben Ezra, B.I. Lembrikov* \\ Faculty of Electrical Engineering, Electronics and Communications \\ Holon Institute of Technology (HIT), 52 Golomb str., P.O.B. 305, \\ Holon 58102, Israel \\ e-mail borisle@hit.ac.il, *Contact person
}

\begin{abstract}
We investigated theoretically the optical injection influence on the dynamics of a quantum dot (QD) in a quantum well (QDWELL) laser. The carrier dynamics is synchronized due to the optical injection, and QDWELL laser manifests a high performance at repetition frequencies up to $25 \mathrm{GHz}$.
\end{abstract}

\section{INTRODUCTION}

A novel type of quantum dot (QD) laser with the-dotin-a-well (DWELL) structure based on InAs QDs grown in the strained InGaAs quantum well $(\mathrm{QW})$ characterized by an extremely low threshold current density of $42.6 \mathrm{~A} \cdot \mathrm{cm}^{-2}$ and a lasing wavelength near $1.3 \mu \mathrm{m}$ has been recently proposed [1]. However, the QDWELL laser modulation bandwidth of $7 \mathrm{GHz}$ is limited by the relaxation oscillation (RO) frequency. In order to improve the performance of directly modulated semiconductor lasers, the optical injection locking (OIL) of semiconductor lasers can be used providing a single-mode regime with side-mode suppression, strongly enhanced RO frequency, enhanced bandwidth, reduced nonlinearity, reduced relative intensity noise (RIN), reduced chirp, increased link gain, and near-single-sideband modulation [2]. The complicated dynamics of optically injected QDWELL lasers has been investigated both theoretically and experimentally using the Lüdge-Schöll (LS) model [3], [4]. We have shown that due to the optical injection, the electron and hole dynamics in QW and QDs is synchronized, and consequently, the QDWELL laser demonstrates a high performance in the large signal regime for the moderate bias current level at the repetition frequencies up to $25 \mathrm{GHz}$.

\section{Theoretical Model of an Optically InJected QDWELL LASER}

The LS rate equation system for the optically injected QDWELL laser with two-level QDs has the form [3].

$$
\begin{gathered}
\frac{d n_{p h}}{d t}=n_{p h}\left[2 \widetilde{W} Z_{a}^{Q D}\left(\rho_{e}+\rho_{h}-1\right)-2 \kappa\right] \\
+\frac{\beta}{A} 2 Z_{a}^{Q D} R_{s p}\left(\rho_{e}, \rho_{h}\right)+\frac{2 K}{\tau_{i n}} \sqrt{n_{p h} n_{p h}^{0}} \cos \left(\Phi-2 \pi \Delta \nu_{i n j} t\right) \\
\frac{d \Phi}{d t}=\frac{\alpha}{2}\left[2 \widetilde{W}\left(\rho_{e}+\rho_{h}-1\right)-2 \kappa\right] \\
+\frac{K}{\tau_{i n}} \sqrt{\frac{n_{p h}^{0}}{n_{p h}}} \sin \left(\Phi-2 \pi \Delta \nu_{i n j} t\right)
\end{gathered}
$$

$$
\begin{gathered}
\frac{d \rho_{e, h}}{d t}=-\widetilde{W} A\left(\rho_{e}+\rho_{h}-1\right) n_{p h}-R_{s p}\left(\rho_{e}, \rho_{h}\right) \\
+S_{e, h}^{i n}\left(w_{e}, w_{h}\right)\left(1-\rho_{e, h}\right)-S_{e, h}^{\text {out }}\left(w_{e}, w_{h}\right) \rho_{e, h} \\
\frac{d w_{e, h}}{d t}=\frac{j}{e_{0}} \\
-2 N^{Q D}\left[S_{e, h}^{\text {in }}\left(w_{e}, w_{h}\right)\left(1-\rho_{e, h}\right)-S_{e, h}^{\text {out }}\left(w_{e}, w_{h}\right) \rho_{e, h}\right]-\widetilde{R}_{s p}
\end{gathered}
$$

where $A$ is the in-plane area of the QW. $\rho_{e, h}$ are the electron and hole occupation probabilities in the confined QD levels, respectively; $w_{e, h}$, are the electron and hole densities in the QW. The laser frequency $\nu$ and the injected light frequency $\nu_{i n j}$ are very close: $\nu_{i n j} / v \approx 1 ; K=\sqrt{T_{i n j} n_{i n j} / n_{p h}^{0}}$ is the injection strength, $T_{i n j}$ is the transmission coefficient of the cavity mirror; $n_{i n j}$ is the injected photon density in the active region of the QD laser; $n_{p h}^{0}$ is the steady-state photon density without injection $(K=0)$. The input detuning $\Delta \nu_{i n j}=\nu_{i n j}-\nu_{L} ; Z_{a}^{Q D}=a_{L} A N_{a}^{Q D}$ is the number of active QDs inside the waveguide, $a_{L}$ is the number of selforganized QD layers; $2 \kappa$ are the optical intensity losses. $\widetilde{W}=$ $|\mu|^{2} \omega_{L} T_{2} /\left(\varepsilon_{0} \varepsilon_{b g} \hbar V^{w}\right)$ and $W=|\mu|^{2} \sqrt{\varepsilon_{b g}} \omega_{L}^{3} /\left(3 \pi \varepsilon_{0} \hbar c^{3}\right)$ are the Einstein coefficients for the coherent interaction and for spontaneous emission resulting from the incoherent interaction of the QD with all resonator modes [3], $\mu$ is the associated dipole moment of the optical transition, $T_{2}$ is the lifetime of the microscopic polarization; $\hbar / T_{2}$ is width of the optical transition; $V^{w}=A h^{w}$ is the volume of the optical waveguide; $\varepsilon_{0}$ is the free space permittivity. The linear gain coefficient for the induced emission and absorption $g=2 \widetilde{W} Z_{a}^{Q D}, \beta$ is the spontaneous emission factor. The spontaneous emission rates in QDs and QW are, respectively, $R_{s p}\left(\rho_{e}, \rho_{h}\right)=W \rho_{e} \rho_{h}$, $\widetilde{R}_{s p}=B^{S} w_{e} w_{h}[3] ; N_{a}^{Q D}$ is the density per unit area of the active QDs of lasing subgroup, $N^{Q D}$ is the density per unit area of all QDs, $\tau_{i n}=2 L \sqrt{\varepsilon_{b g}} / c$ is the time for one round trip of the light in the cavity of length $L ; \varepsilon_{b g}$ is the static relative permittivity of the background medium, $c$ is the speed of light in vacuum, $L$ is the cavity length; $j$ is the injection current density, $e_{0}$ is the elementary charge, $B^{S}$ is the band-band recombination coefficient; $\alpha$ is the linewidth enhancement factor (LEF). $S_{e}^{\text {out }}, S_{h}^{\text {out }}, S_{e}^{\text {in }}, S_{h}^{\text {in }}$ are the scattering rates of electrons and holes for scattering in and out of QDs. Their explicit expressions and numerical values of the coefficients 

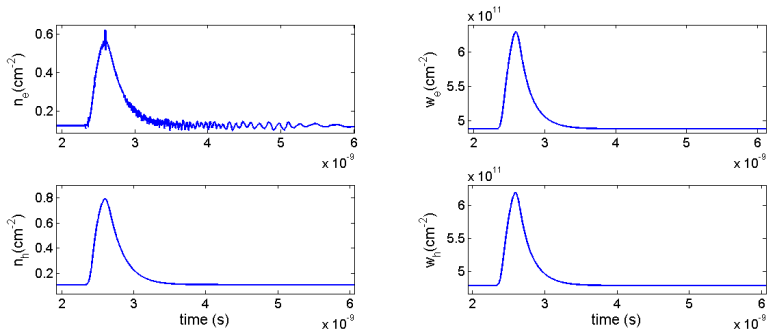

Fig. 1. QD and QW carrier switching dynamics without detuning, the high level of optical pumping $P_{o p t} \gtrsim 1 \mathrm{~mW}$, and the bias cuurent density $j=$ $3 j_{t h}$

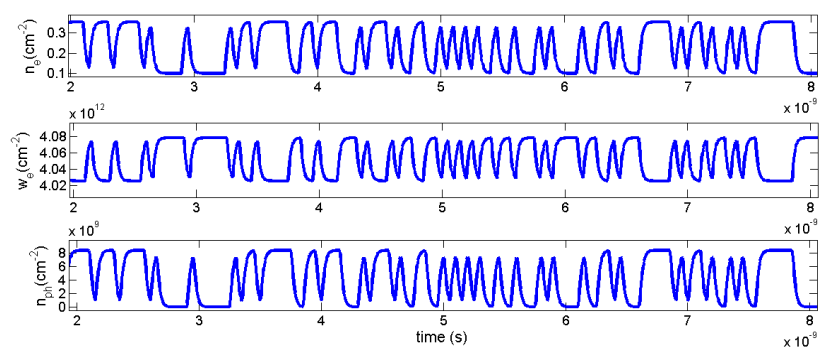

Fig. 2. The large signal QD $n_{e} / N^{Q D}=\rho_{e}$ and QW $w_{e}$ electron dynamics and the output photon density $n_{p h}$ for the high level of optical injection power $P_{\text {opt }} \gtrsim 1 \mathrm{~mW}$

can be found in [3]. The corresponding electron and hole lifetimes $\tau_{e, h}$ are given by: $\tau_{e, h}=\left(S_{e, h}^{\text {out }}+S_{e, h}^{i n}\right)^{-1}$ [3].

\section{Simulation Results}

We solved numerically equations (1)-(4) for the the turnon dynamics and the large signal response of the optically injected QDWELL laser using the typical values of QDWELL laser parameters [3]. The simulation results are presented in Figs. 1-4. It is seen from Fig. 1 that for the moderate bias current density $j=3 j_{t h}$, without detuning and with the injected optical power $P_{\text {opt }} \sim 1 \mathrm{~mW}$ the QD and QW carrier pulses are synchronized. The numerical simulations show that for larger bias current densities of $(5-6) j_{t h}$, the detuning of $1-5 \mathrm{GHz}$, and the injected optical power up to $3-4 \mathrm{~mW}$, the switching in the turn-on regime is accompanied by the rapid oscillations of the QDWELL laser output photon density which corresponds to the experimental results [5].

The simulation results for the large signal response of the optically injected QDWELL laser are shown in Figs. 2 and 3. The electrical PRBS signal with a length of $\left(2^{13}-1\right)$, and the optical injection power $P_{o p t} \gtrsim 1 \mathrm{~mW}$ have been used. Due to the optical injection, the output optical pulses strictly follow the QW and QD synchronized electron and hole density oscillations, unlike the case without the optical injection where both the simulated and measured data for the laser output differ significantly from the pump-current signal. Under the optical injection, the radiative transition rates in QDs are determined mainly by the optical injection power and the frequency detuning between the master and the slave lasers. In our case of the electrical PRBS input signal, due to

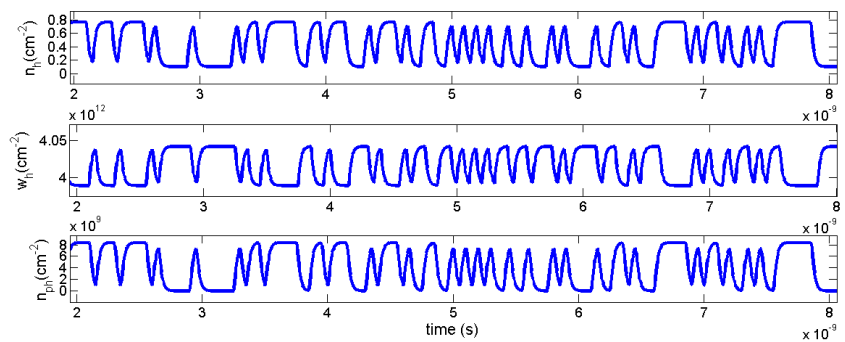

Fig. 3. The large signal QD $n_{h} / N^{Q D}=\rho_{h}$ and QW $w_{h}$ hole dynamics and the output photon density $n_{p h}$ for the high level of optical injection power $P_{\text {opt }} \gtrsim 1 m W$

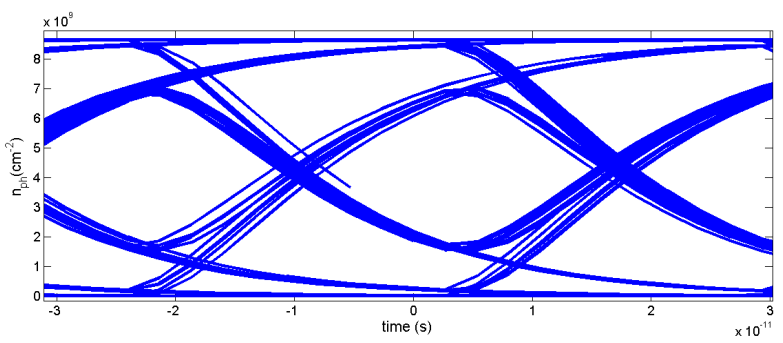

Fig. 4. Eye diagram for the repetition rate of $25 \mathrm{GHz}$, optical injection power $P_{\text {opt }} \gtrsim 1 \mathrm{~mW}$, and large signal

the optical injection enhanced RO frequency, the eye diagram manifests a good performance even at the repetition frequency of $25 \mathrm{GHz}$ as it is seen in Fig. 4 .

\section{Conclusion}

We investigated theoretically the optical injection influence on the turn-on dynamics and the large signal response of the electron and hole densities $n_{e, h}$ in QDs and $w_{e, h}$ in QW of the QDWELL laser, and on its output photon density $n_{p h}$ by solving numerically the LS rate equation system (1)(4). The optical injection results in a synchronized behavior of electrons and holes in QDs and QW for both the turnon regime and the large signal response. As a result, the QDWELL laser performance improves up to the repetition frequency of $25 \mathrm{GHz}$.

\section{REFERENCES}

[1] A. Stintz, G.T. Liu, H. Li, L.F. Lester, and K.J. Malloy, "Low-threshold current density $1.3-\mu m$ InAs quantum dot lasers with the dots-in-well (DWELL) structure", IEEE Photonic Technology Letters, vol. 12, no. 6, pp. 591-593, June 2000.

[2] E. K. Lau, Liang Jie Wong, and Ming C. Wu, "Enhanced modulation characteristics of injection-locked lasers: a tutorial", IEEE Journal of Selected Topics in Quantum Electronics, vol. 15, no. 3, pp. 618-633, May/June 2009.

[3] J. Pausch, C. Otto, E. Tylaite, N. Majer, E. Schöll and K. Lüdge, "Optically injected quantum dot lasers: impact of nonlinear carrier lifetimes on frequency-locking dynamics", New Journal of Physics, vol. 14, pp. 1-20, 2012

[4] Y. Ben Ezra, B.I. Lembrikov, "Synchronized Carrier Dynamics in Quantum Dot-in-a-Well (QDWELL) Laser under an Optical Injection", IEEE Journal of Selected Topics in Quantum Electronics, 2013, Accepted for publication

[5] D. Goulding, S.P. Hegarty, O. Rasskazov, S.Melnik, M. Hartnett, G. Greene, J.G. McInerney, D. Rachinskii, and G. Huyer, "Excitability in a quantum dot laser with optical injection", Physical Review Letters, vol. 98, no. 15, pp. 153903-1-4, April 2007. 